Journal of Agricultural Sciences
(Tarim Bilimleri Dergisi)

\title{
Biochemical Composition and Antioxidant Activity of Different Types of Tomatoes Affected by Ethylene Treatment
}

\author{
Qasid ALI $^{\mathrm{I}} \mathbb{D}$, Mehmet Seckin KURUBAS ${ }^{\mathrm{a}} \mathbb{D}$, Mustafa ERKAN ${ }^{\mathrm{a} *} \mathbb{D}$

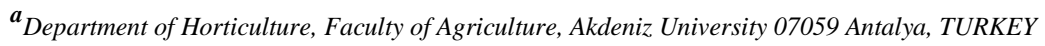 \\ ARTICLE INFO \\ Research Article \\ Corresponding Author: Mustafa ERKAN, E-mail: erkan@akdeniz.edu.tr \\ Received: 04 May 2020 / Revised: 11 August 2020 / Accepted: 21 September 2020 / Online: 20 January 2022
}

\section{ABSTRACT}

The effect of ethylene on biochemical composition and antioxidant activity in beefsteak, heirloom and cluster type of tomatoes were determined. For that purpose, tomato fruit were harvested at breaker maturity stage and divided into two groups one of which was applied with $150 \mu \mathrm{L} \mathrm{L} \mathrm{L}^{-1}$ ethylene while another remained untreated. Ethylene treated and untreated control fruit were stored at $12{ }^{\circ} \mathrm{C}$ and $90+5 \%$ relative humidity for 35 days with subsamples removed every 7 days for quality analysis. After each removal time, fruit were kept at $20^{\circ} \mathrm{C}$ for additional 3 days to determine shelf life performance. Ethylene treatment enhanced the breakdown of total chlorophyll and accumulation of lycopene and carotenoid contents. At the end of cold storage and shelf life period, the maximum antioxidant activity, carotenoid and flavonoid contents were recorded in ethylene treated heirloom type tomatoes. It can be concluded that ethylene treated heirloom type tomatoes exhibited maximal postharvest quality as compared to beefsteak and cluster type of tomato in term of biochemical composition and antioxidant activity after 35 days of cold storage and shelf life.

Keywords: Ethylene, Antioxidant, Cold storage, Shelf life, Tomato quality

(c) Ankara University, Faculty of Agriculture

\section{Introduction}

Tomatoes are vital part of human nutrition around the world. Scientific studies have shown that tomatoes contain high amount of carotenoid, antioxidant, lycopene and are associated with dietary intake that reduces the risk of chronic diseases, cancer, osteoporosis and cardiovascular diseases in humans (Rao et al. 1998; Frusciante et al. 2007; Bhowmik et al. 2012). The presence of carotenoids, especially lycopene, ascorbic acid, vitamin E, phenolic compounds and different antioxidant properties in tomatoes affect the human health (Frusciante et al. 2007; Bhowmik et al. 2012).

Ripening is genetically programmed process which show substantial changes in color, texture, flavor and aroma (Alexander \& Grierson 2002). Tomatoes being a climacteric fruit are sensitive to ripening hormone ethylene. Exogenous application of ethylene in climacteric fruit can trigger and enhance the ripening process (Tucker 1993). It is thought that ethylene regulates the formation of carotenoids present in the chloroplast through synthesis of new enzymes and influence mitochondria based organic acid concentrations (Mcglasson 1970). Ethylene does not only affect biochemical composition but also increases respiration rate and aging of fruit and vegetables (Prasanna et al. 2007).

The higher antioxidant activity of tomato could mitigate the effect of ethylene as ripening process involved series of physiological and biochemical changes in which antioxidant properties play fundamental role (Jimenez et al. 2002). The signal transduction by ethylene in important for secondary metabolites synthase. The biosynthesis of flavanol is modulated by ethylene through transcription factor (Lewis et al. 2011). During stress the polyphenol oxidase (PPO) activity is increased through ethylene signalling which supports its involvement in the defence resistance of plant (Bosch et al. 2014). In tea, the phenolic compounds, flavonoids and antioxidant activity were increased by ethylene signalling (Ke et al. 2018).

The nutritional value, color and flavor of tomatoes are mainly dependent on the ratios of lycopene, $\beta$-carotene, ascorbic acid and sugars (Nguyen \& Schwartz 1999). Epidemiological studies have shown that lycopene and $\beta$-carotene serve as an antioxidant and functional food (Tonucci et al. 1995). The assessment of the effects of different tomato varieties on the synthesis of carotenoid and other phenolic compounds are important to enhance the concentration of these antioxidant compounds. The studies conducted previously confirmed that amount of carotenoid and other antioxidant compounds in tomato fruit exhibit differences among genotypes (George et al. 2004). According to Viskelis et al. (2007) the Lithuanian cultivar 'Rutuliai' displayed

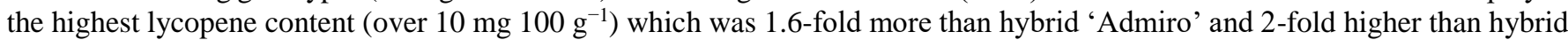


'Kassa'. Radzevicius et al. (2013) reported that the different cultivars of tomato have wide variations in term of ascorbic acid contents. The increasing economic importance of tomato throughout the world as a functional food have necessitated to find out the effect of ethylene on the biochemical composition and antioxidant capacity of different types of tomato and therefore this experiment was conducted.

\section{Material and Methods}

Beefsteak (cv. Tybif), heirloom (cv. Yuksel Koy) and cluster (cv. Merkur) types of tomato were harvested at 'breaker stage'. There was definite break in color from green to tannish yellow, pink or red on not more than $10 \%$ of the surface. All fruit were picked from a commercial greenhouse in Antalya, Turkey (36 59'57.3” N and 30 51'20.4” E). During the entire vegetation period, uniform irrigation and fertigation management procedures were applied to the tested tomato types. All fruit were harvested on the same day and immediately brought to the postharvest physiology laboratory at Akdeniz University, Antalya, Turkey. Fruit with any defects i.e. decayed, bruised and non-uniform, were discarded and the remainder were split into two groups. The first group of tomato fruit were applied with $150 \mu \mathrm{L} \mathrm{L}^{-1}$ of ethylene at $20{ }^{\circ} \mathrm{C}$ in a $20 \mathrm{~m}^{3}$ room and the second group were left untreated (control). Both groups of fruit samples were stored at $12^{\circ} \mathrm{C}$ and $90 \pm 5 \%$ relative humidity for 35 days. Fruit samples for different quality analysis were removed from cold room at 7 days intervals and they were also kept at $20{ }^{\circ} \mathrm{C}$ and $60 \pm 5 \%$ relative humidity for additional 3 days to simulate shelf life performance.

Tomato puree was utilized for analysis of total chlorophyll, lycopene, total phenolic, carotenoid, flavonoid, ascorbic acid contents and antioxidant activity. Homogenization of tomato samples for all quality analysis ultra turrax homogenizer (IKALabortechnique Typ T 25 JANKE \& KUNKEL GMBH \& CO.KG) was used. The samples absorbances for all quality analysis were read through Analytik Jena AG Specord 40 ST spectrophotometer.

The total chlorophyll contents were determined according to the method of Lichtenthaler \& Wellburn (1983). Tomato puree of $3 \mathrm{~g}$ was homogenized with $80 \%$ acetone through ultra turrax homogenizer. The centrifuge of homogenized samples was performed at $8600 \mathrm{x}$ g for 5 min under $4{ }^{\circ} \mathrm{C}$. After centrifuge, sample supernatant was used for determination of chlorophyll content. The supernatant was read through Specord 40 ST spectrophotometer against blank $80 \%$ acetone solvent at the wavelengths of 646 and $663 \mathrm{~nm}$. The total chlorophyll contents of tomato fruit were computed through equation (1) given below and given as $\mathrm{g} \mathrm{kg}^{-1}$ fresh weight (fw).

Chlorophyll $\mathrm{a}=12.21 \times \mathrm{A}_{663}-2.81 \times \mathrm{A}_{646}$

Chlorophyll $\mathrm{b}=20.13 \times \mathrm{A}_{646}-5.03 \times \mathrm{A}_{663}$

Total chlorophyll $=\left(\mathrm{C}_{\mathrm{a}}+\mathrm{C}_{\mathrm{b}}\right)$

The method explained by Fish et al. (2002) was used for the determination of lycopene contents in different types of tomato. For that purpose, the tomato samples were homogenized through homogenizer and $0.5 \mathrm{~g}$ of samples were weighed and put in 50 $\mathrm{mL}$ test tubes. $5 \mathrm{~mL}$ butylated hydroxytoluene (BHT) prepared with acetone $(0.05 \% \mathrm{w} / \mathrm{v}), 5 \mathrm{~mL}$ ethanol $(95 \%)$ and hexane at 10 $\mathrm{mL}$ concentration were added to sample. Prepared samples were shaken at $4{ }^{\circ} \mathrm{C}$ for 5 min at 180 rpm through shaker. After, 3 $\mathrm{mL}$ distilled water was added to sample and shaken again for $5 \mathrm{~min}$. Then, the samples were left to separate the phase for $5 \mathrm{~min}$ at room temperature to obtain colored layer of hexane at the top surface. The supernatant containing hexane layer was read in spectrophotometer at $563 \mathrm{~nm}$ of absorbance. Data obtained from the measurements were calculated by equation (2) below and reported as $\mathrm{mg} \mathrm{kg}^{-1} \mathrm{fw}$.

Lycopene $\left(\mathrm{mg} \mathrm{kg}^{-1}\right)=\mathrm{A}_{503} \times 0.0312 / \mathrm{kg}$, sample

$\mathrm{A}_{503}=$ The absorbance value at $503 \mathrm{~nm}$

$0.0312(\varepsilon)=$ Extinction coefficient of lycopene.

The extraction of fruit samples was done with $80 \%$ methanol for antioxidant activity, total phenolic and total flavonoid contents analysis. For this purpose, tomato puree of $20 \mathrm{~g}$ and $80 \%$ methanol of $20 \mathrm{~mL}$ was homogenized with the help of UltraTurrax homogenizer. The samples were centrifuged at $8600 \mathrm{x} \mathrm{g}$ for $20 \mathrm{~min}$ at $4{ }^{\circ} \mathrm{C}$ after homogenization. The antioxidant activity of tomato was determined according to DPPH method described by Benvenuti et al. (2004). For that purpose, 1 m $M$ DPPH* radical solution of $600 \mu \mathrm{L}$ was taken in 4 test tubes and 1, 2, 3 and $4 \mathrm{~mL}$ of extracted samples were added into test tubes. After, $80 \%$ methanol was used to bring total volume in each tube to $6 \mathrm{~mL}$. The mixture in tubes were vortexed and left to incubate in dark at room temperature for $15 \mathrm{~min}$. Additionally, the control sample was prepared by taking $600 \mu \mathrm{L}$ of $1 \mathrm{~m} M \mathrm{DPPH}$ radical solution and $5.4 \mathrm{~mL}$ of methanol in a tube and allowed to incubate in a dark place at room temperature for 15 min. After incubation samples absorbance were read at $517 \mathrm{~nm}$ wavelength by using spectrophotometer against blank solvent of $80 \%$ methanol and control sample. Percent inhibition values proportionate to each sample volume were computed by using equation (3).

$\%$ inhibition $=\mathrm{A}_{\mathrm{DPPH}}-\mathrm{A}_{\text {Extract }} / \mathrm{A}_{\mathrm{DPPH}} \mathrm{x} 100$

$A_{D P P H}$ DPPH control sample absorbance value; $A_{\text {Extract: }}$ Test sample absorbance value 
The inhibition values and sample volumes were used to obtain a graph. Linear regression analysis was applied to the graph, sample curve and equation explaining the curve was acquired. Equation was used for calculation of $\mathrm{EC}_{50}$ (effective concentration) value of the sample. The antioxidant activity by $\mathrm{DPPH}$ method is determined through $\mathrm{EC}_{50}$ value. The $\mathrm{EC}_{50}$ value reflects amount of antioxidant substances present in fruit and vegetables sample that inhibit $50 \%$ of DPPH radical. Decrease in $\mathrm{EC}_{50}$ value exhibit increase in the antioxidant activity (Cemeroglu 2010). The $\mathrm{EC}_{50}$ value was reported in $\mathrm{g} \mathrm{kg}^{-1} \mathrm{fw} \mathrm{EC}_{50}$.

The total carotenoid content analysis was performed by using the method of Witham et al. (1971). For that purpose, tomato puree of $0.25 \mathrm{~g}$ was homogenized with $10 \mathrm{~mL} 80 \%$ acetone for 3-4 min through ultra-turrax homogenizer and $80 \%$ acetone solvent was used to bring the total volume of sample to $15 \mathrm{~mL}$. After, the samples were centrifuged at $8600 \mathrm{x} \mathrm{g}$ for $10 \mathrm{~min}$ at $4{ }^{\circ} \mathrm{C}$ after homogenization. The carotenoids content was determined by using the supernatant fraction. The samples absorbance used for chlorophyll a, chlorophyll b and carotenoid were $663 \mathrm{~nm}, 645 \mathrm{~nm}$ and $440 \mathrm{~nm}$ respectively. The samples absorbance was read through spectrophotometer against a blank solvent of $80 \%$ acetone. The total carotenoid content was calculated through equation (4) and expressed as $\mathrm{g} \mathrm{kg}^{-1} \mathrm{fw}$.

Chlorophyll a $\left(\mathrm{g} \mathrm{kg}^{-1}\right)=\left[12.7\right.$ (D663) - 2.69 (D645)] x V/1000 x W Chlorophyll b $\left(\mathrm{g} \mathrm{kg}^{-1}\right)=[22.9(\mathrm{D} 645)-4.68$ (D663) $] \times \mathrm{V} / 1000 \times \mathrm{W}$

Carotenoids $\left(\mathrm{g} \mathrm{kg}^{-1}\right)=[4.69(\mathrm{D} 440)-($ chlorophyll a + chlorophyll b $) \times 0.286] \times \mathrm{V} / 1000 \times \mathrm{W}$

$\mathrm{V}=$ Extract volume

$\mathrm{W}=$ Sample quantity

The total flavonoid content of tomatoes was analysed through the procedure explained by Karadeniz et al. (2005). In $50 \mathrm{~mL}$ tube, $1 \mathrm{~g}$ of tomato puree, distilled water of $5 \mathrm{~mL}$ and $5 \% \mathrm{NaNO}_{2}(\mathrm{Merck})$ of $0.3 \mathrm{~mL}$ were added, respectively. The test tubes were closed and strongly mixed. 5 min later $0.6 \mathrm{~mL}$ of $10 \% \mathrm{AlCl}_{3} .6 \mathrm{H}_{2} \mathrm{O}$ (Merck) was added and after $5 \mathrm{~min} 2 \mathrm{~mL}$ of $1 \mathrm{~mol} \mathrm{~L}-1$ $\mathrm{NaOH}$ was added. The distilled water was used to bring total volume in tube to $10 \mathrm{~mL}$. Tubes were then vortexed and samples absorbance was measured at wavelength of $510 \mathrm{~nm}$ using spectrophotometer against solvent of blank $80 \%$ methanol. The standard calibration curve prepared with catechin were used to compute the total flavonoid content of tomatoes and given as mg $\mathrm{kg}^{-1} \mathrm{fw}$.

The total phenolic contents analysis was performed according to the Folin-Ciocalteu method explained by Spanos and Wrolstad (1990). Extract of $0.1 \mathrm{~mL}$ was blended with distilled water of $0.9 \mathrm{~mL}$ and $0.2 \mathrm{~mol} \mathrm{~L}{ }^{-1} \mathrm{~N}$ Foline-Ciocalteu reagent of 5 $\mathrm{mL}$. After $3 \mathrm{~min}$, aqueous solution of $\mathrm{Na}_{2} \mathrm{CO}_{3}\left(75 \mathrm{~g} \mathrm{~L}^{-1}\right)$ at $4 \mathrm{~mL}$ concentration was added into blend and samples were kept for $2 \mathrm{~h}$ in the dark at room temperature. The samples absorbance was recorded at the wavelength of $765 \mathrm{~nm}$ against blank $80 \%$ methanol solvent through spectrophotometer. The total phenolic contents calculated were reported as mg of gallic acid equivalent per $\mathrm{kg}\left(\mathrm{mg} \mathrm{kg}^{-1} \mathrm{GAE}\right) \mathrm{fw}$.

The total ascorbic acid contents analysis was conducted according to Cemeroglu (2010). For that purpose, the tomato samples were extracted with $6 \%$ metaphosphoric acid and in $50 \mathrm{~mL}$ tube, extract of $5 \mathrm{~mL}$, acetate buffer solution (pH 4.0$)$ of $5 \mathrm{~mL}, 2,6$ dichlorophenolindophenol dye solution of $1 \mathrm{~mL}$, and xylene of $10 \mathrm{~mL}$ were added. After, the tubes were stirred for $10 \mathrm{~s}$ and centrifugation was performed at $8600 \mathrm{x}$ g for $10 \mathrm{~min}$ at $4{ }^{\circ} \mathrm{C}$. The control sample was prepared in a test tube containing $6 \%$ metaphosphoric acid of $5 \mathrm{~mL}$, acetate buffer solution ( $\mathrm{pH} 4.0)$ of $5 \mathrm{~mL}, 1 \mathrm{~mL}$ of 2,6 dichlorophenolindophenol dye solution and $10 \mathrm{~mL}$ of xylene. Samples absorbance was recorded at wavelength of $500 \mathrm{~nm}$ through spectrophotometer against xylene and control sample. The ascorbic acid calibration curve equation was $y=0.0123 x+0.0134$ and coefficient of determination was $R^{2}$

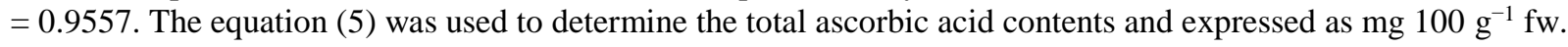

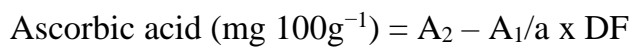

$\mathrm{A}_{1}$ : Extract sample absorbance value; $\mathrm{A}_{2}$ : Control sample absorbance value; DF: Dilution factor; a: Ascorbic acid standard curve slope

The experiment was designed according to completely randomized design (CRD) with three replications. Each replication contained ten fruit. Duncan's multiple range test was used to determine significant differences among means. Mean values obtained were analysed with SAS program.

\section{Results and Discussion}

\subsection{Total chlorophyll content}

Ethylene treated tomatoes had less chlorophyll content and different types of tomatoes exhibited decrease in total chlorophyll content by the end of storage period. After cold storage, the maximum amount of total chlorophyll content $\left(0.0030 \mathrm{~g} \mathrm{~kg}^{-1}\right) \mathrm{was}$ found in untreated heirloom type while the minimum total chlorophyll content $\left(0.0001 \mathrm{~g} \mathrm{~kg}^{-1}\right)$ was obtained in ethylene treated cluster type of tomatoes (Table 1). There were no significant differences between ethylene treated heirloom and cluster type of 
tomatoes by the end of cold storage. After shelf life period, the highest total chlorophyll content $\left(0.0016 \mathrm{~g} \mathrm{~kg}^{-1}\right)$ was noted in control beefsteak type while lowest total chlorophyll content $\left(0.0004 \mathrm{~g} \mathrm{~kg}^{-1}\right)$ was obtained in ethylene treated cluster type of tomatoes (Table 2). There were no significant differences between heirloom and cluster type of tomatoes at the end of shelf life period.

Table 1- Effect of ethylene on the total chlorophyll, lycopene, total phenolic contents, antioxidant activity, carotenoid, flavonoid, and ascorbic acid contents of different types of tomatoes under cold storage at $12{ }^{\circ} \mathrm{C}$

\begin{tabular}{|c|c|c|c|c|c|c|c|}
\hline \multirow[b]{2}{*}{ Parameters } & \multirow[b]{2}{*}{ Treatments } & \multicolumn{6}{|c|}{ Storage duration (Days) } \\
\hline & & $\mathbf{0}$ & 7 & 14 & 21 & 28 & 35 \\
\hline \multirow{7}{*}{$\begin{array}{l}\text { Total chlorophyll content } \\
\left(\mathrm{g} \mathrm{kg}^{-1} \text { fw) }\right.\end{array}$} & $\mathbf{B S}^{\dagger}$ & $0.050 \mathrm{ab} *$ & $0.0042 \mathrm{ac}$ & $0.0024 \mathrm{cj}$ & $0.0017 \mathrm{dj}$ & $0.0011 \mathrm{gj}$ & $0.0010 \mathrm{gj}$ \\
\hline & BS+Ethyl. & $0.0038 \mathrm{af}$ & 0.0030bi & $0.0021 \mathrm{cj}$ & $0.0018 \mathrm{di}$ & $0.0014 \mathrm{fj}$ & 0.0007hj \\
\hline & HL & $0.0044 \mathrm{ac}$ & $0.0039 \mathrm{ae}$ & $0.0038 \mathrm{ae}$ & $0.0038 \mathrm{af}$ & $0.0032 \mathrm{bg}$ & 0.0030bi \\
\hline & HL+Ethyl. & $0.0040 \mathrm{ad}$ & $0.0037 \mathrm{bf}$ & $0.0031 \mathrm{bh}$ & $0.0029 \mathrm{bi}$ & $0.0012 \mathrm{gj}$ & $0.0005 \mathrm{j}$ \\
\hline & CL & $0.0061 \mathrm{a}$ & $0.0042 \mathrm{ac}$ & $0.0033 \mathrm{bg}$ & $0.0033 \mathrm{bg}$ & $0.0030 \mathrm{bi}$ & $0.0012 \mathrm{gj}$ \\
\hline & CL+Ethyl. & $0.0053 \mathrm{ab}$ & $0.0021 \mathrm{cj}$ & $0.0016 \mathrm{ej}$ & $0.0008 \mathrm{hj}$ & $0.0007 \mathrm{ij}$ & $0.0001 \mathrm{j}$ \\
\hline & \multicolumn{7}{|c|}{ 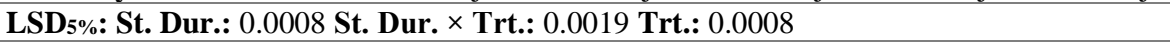 } \\
\hline \multirow{7}{*}{$\begin{array}{l}\text { Lycopene content } \\
\left(\mathrm{mg} \mathrm{kg}^{-1} \text { fw) }\right.\end{array}$} & BS & $1.93 \mathrm{k}$ & $6.47 \mathrm{ik}$ & $9.96 \mathrm{gk}$ & $17.50 \mathrm{eh}$ & $26.12 \mathrm{de}$ & $25.84 \mathrm{de}$ \\
\hline & BS+Ethyl. & $5.10 \mathrm{ik}$ & $6.62 \mathrm{ik}$ & $12.63 \mathrm{fk}$ & $18.11 \mathrm{eg}$ & $22.84 \mathrm{df}$ & $26.65 \mathrm{de}$ \\
\hline & HL & $2.98 \mathrm{jk}$ & $5.131 \mathrm{ik}$ & $14.26 \mathrm{fj}$ & $18.59 \mathrm{eg}$ & $39.08 \mathrm{ac}$ & $45.25 \mathrm{a}$ \\
\hline & HL+Ethyl. & $4.07 \mathrm{ik}$ & $5.14 \mathrm{ik}$ & $10.15 \mathrm{gk}$ & 31.06bd & $42.95 \mathrm{a}$ & $47.37 \mathrm{a}$ \\
\hline & $\mathbf{C L}$ & $2.31 \mathrm{k}$ & $2.46 \mathrm{k}$ & 6.76hk & $25.57 \mathrm{de}$ & $38.18 \mathrm{ac}$ & $40.30 \mathrm{ac}$ \\
\hline & CL+Ethyl. & $2.98 \mathrm{jk}$ & $4.87 \mathrm{ik}$ & $14.81 \mathrm{fi}$ & 26.59de & $30.02 \mathrm{~cd}$ & $41.46 \mathrm{ab}$ \\
\hline & \multicolumn{7}{|c|}{ LSD5\%: St. Dur.: 3.825 St. Dur. $\times$ Trt.: 9.3694 Trt.: 3.825} \\
\hline \multirow{7}{*}{$\begin{array}{l}\text { Antioxidant Activity } \\
\left(\mathrm{g} \mathrm{kg}^{-1} \text { fw EC } \mathrm{C}_{50}\right)\end{array}$} & BS & $1.53 \mathrm{a}$ & $0.75 \mathrm{di}$ & $1.09 \mathrm{bd}$ & $0.56 \mathrm{fl}$ & $0.66 \mathrm{el}$ & $0.75 \mathrm{di}$ \\
\hline & BS+Ethyl. & $1.40 \mathrm{ab}$ & $1.14 \mathrm{bc}$ & $0.72 \mathrm{dj}$ & $0.72 \mathrm{dj}$ & $0.85 \mathrm{cg}$ & $0.89 \mathrm{cf}$ \\
\hline & HL & $0.44 \mathrm{gl}$ & $0.36 \mathrm{il}$ & 0.251 & $0.27 \mathrm{kl}$ & $0.47 \mathrm{gl}$ & $0.43 \mathrm{hl}$ \\
\hline & HL+Ethyl. & $0.30 \mathrm{kl}$ & $0.27 \mathrm{kl}$ & $0.66 \mathrm{ek}$ & $0.32 \mathrm{jl}$ & $0.42 \mathrm{hl}$ & $0.33 \mathrm{jl}$ \\
\hline & CL & $1.02 \mathrm{ce}$ & $0.79 \mathrm{ch}$ & $0.52 \mathrm{fl}$ & $0.62 \mathrm{el}$ & $0.83 \mathrm{ch}$ & $0.65 \mathrm{el}$ \\
\hline & CL+Ethyl. & $1.50 \mathrm{a}$ & $0.77 \mathrm{ci}$ & $0.59 \mathrm{fl}$ & $0.67 \mathrm{ek}$ & $0.72 \mathrm{dj}$ & $0.65 \mathrm{el}$ \\
\hline & \multicolumn{7}{|c|}{ LSD5\%: St. Dur.: 0.1369 St. Dur. $\times$ Trt.: 0.3353 Trt.: 0.1369} \\
\hline \multirow{7}{*}{$\begin{array}{l}\text { Carotenoid content } \\
\left(\mathrm{g} \mathrm{kg}^{-1} \mathrm{fw}\right)\end{array}$} & BS & $0.0046 \mathrm{jk}$ & $0.0062 \mathrm{ik}$ & $0.0084 \mathrm{hk}$ & $0.0113 \mathrm{gk}$ & $0.0201 \mathrm{eg}$ & $0.0287 \mathrm{de}$ \\
\hline & BS+Ethyl. & $0.0042 \mathrm{jk}$ & $0.0116 \mathrm{gk}$ & $0.0149 \mathrm{fj}$ & $0.0155 \mathrm{fj}$ & $0.0231 \mathrm{ef}$ & $0.0680 \mathrm{~b}$ \\
\hline & HL & $0.0048 \mathrm{jk}$ & $0.0049 \mathrm{jk}$ & $0.0050 \mathrm{jk}$ & $0.0112 \mathrm{gk}$ & $0.0156 f j$ & $0.0172 \mathrm{fi}$ \\
\hline & HL+Ethyl. & $0.0045 \mathrm{jk}$ & $0.0058 \mathrm{ik}$ & $0.0062 \mathrm{ik}$ & $0.080 \mathrm{hk}$ & $0.0142 \mathrm{fj}$ & $0.1182 \mathrm{a}$ \\
\hline & CL & $0.0020 \mathrm{k}$ & $0.0046 \mathrm{jk}$ & $0.0051 \mathrm{jk}$ & $0.0068 \mathrm{ik}$ & $0.0149 \mathrm{fj}$ & $0.0250 \mathrm{df}$ \\
\hline & CL+Ethyl. & $0.0051 \mathrm{jk}$ & $0.0099 \mathrm{gk}$ & $0.0148 \mathrm{fj}$ & $0.0185 \mathrm{eh}$ & $0.0340 \mathrm{~d}$ & $0.0545 c$ \\
\hline & \multicolumn{7}{|c|}{ LSD5\%: St. Dur.: 0.0039 St. Dur. $\times$ Trt.: 0.0095 Trt.: 0.0039} \\
\hline \multirow{7}{*}{$\begin{array}{l}\text { Flavonoid content } \\
\left(\mathrm{mg} \mathrm{kg}^{-1} \mathrm{fw}\right)\end{array}$} & BS & $252.7 \mathrm{a}$ & $709.7 \mathrm{~cd}$ & $57.6 \mathrm{~cd}$ & $56.3 \mathrm{~cd}$ & $25.2 d$ & $9.8 \mathrm{~d}$ \\
\hline & BS+Ethyl. & $237.2 \mathrm{ab}$ & 123.9ad & $65.8 \mathrm{~cd}$ & $61.9 \mathrm{~cd}$ & $37.2 \mathrm{~cd}$ & $22.6 \mathrm{~d}$ \\
\hline & HL & $113.5 \mathrm{bd}$ & $72.7 \mathrm{~cd}$ & $40.0 \mathrm{~cd}$ & $34.7 \mathrm{~cd}$ & $29.4 d$ & $23.0 \mathrm{~d}$ \\
\hline & HL+Ethyl. & $106.0 \mathrm{bd}$ & $77.6 \mathrm{~cd}$ & $59.1 \mathrm{~cd}$ & $47.4 \mathrm{~cd}$ & $45.5 \mathrm{~cd}$ & $42.2 \mathrm{~cd}$ \\
\hline & $\mathbf{C L}$ & $133.2 \mathrm{ad}$ & $60.8 \mathrm{~cd}$ & $52.0 \mathrm{~cd}$ & $33.0 \mathrm{~d}$ & $30.1 \mathrm{~d}$ & $12.3 \mathrm{~d}$ \\
\hline & CL+Ethyl. & $183.5 \mathrm{ac}$ & 101.6bd & $55.5 \mathrm{~cd}$ & $41.7 \mathrm{~cd}$ & $32.7 \mathrm{~d}$ & $27.5 \mathrm{~d}$ \\
\hline & \multicolumn{7}{|c|}{ 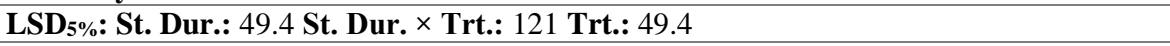 } \\
\hline \multirow{7}{*}{$\begin{array}{l}\text { Total phenolic contents } \\
\left(\mathrm{mg} \mathrm{kg}^{-1} \text { GAE fw) }\right.\end{array}$} & BS & 26.0be & $20.6 \mathrm{cg}$ & $20.0 \mathrm{cg}$ & $16.3 \mathrm{dg}$ & $16.2 \mathrm{dg}$ & $13.7 \mathrm{fg}$ \\
\hline & BS+Ethyl. & $24.2 \mathrm{bf}$ & $22.4 \mathrm{bf}$ & $21.6 \mathrm{cf}$ & $19.7 \mathrm{cg}$ & $17.1 \mathrm{dg}$ & $12.8 \mathrm{fg}$ \\
\hline & HL & $33.4 \mathrm{ab}$ & $30.1 \mathrm{ac}$ & $21.1 \mathrm{cf}$ & $20.0 \mathrm{cg}$ & $18.7 \mathrm{cg}$ & $16.2 \mathrm{dg}$ \\
\hline & HL+Ethyl. & $37.2 \mathrm{a}$ & 26.2bd & $23.4 \mathrm{bf}$ & $23.4 \mathrm{bf}$ & $17.1 \mathrm{dg}$ & $17.1 \mathrm{dg}$ \\
\hline & $\mathbf{C L}$ & $21.0 \mathrm{cf}$ & $19.7 \mathrm{cg}$ & $19.3 \mathrm{cg}$ & $14.7 \mathrm{dg}$ & $13.3 \mathrm{fg}$ & $9.0 \mathrm{~g}$ \\
\hline & CL+Ethyl. & $23.1 \mathrm{bf}$ & $21.8 \mathrm{cf}$ & $21.2 \mathrm{cf}$ & $18.9 \mathrm{cg}$ & $16.8 \mathrm{dg}$ & $14.4 \mathrm{eg}$ \\
\hline & \multicolumn{7}{|c|}{ LSD 5 : St. Dur.: 3.9 St. Dur. × Trt.: 9.5 Trt.: 3.9} \\
\hline \multirow{6}{*}{$\begin{array}{l}\text { Ascorbic acid } \\
\left(\mathbf{m g} \mathbf{1 0 0 g}^{-1} \text { fw }\right)\end{array}$} & BS & $32.17 \mathrm{a}$ & $22.90 \mathrm{dj}$ & $23.57 \mathrm{cg}$ & 19.5.fk & $19.52 \mathrm{fk}$ & $17.18 \mathrm{k}$ \\
\hline & BS+Ethyl. & $29.55 \mathrm{ab}$ & $23.41 \mathrm{ch}$ & $19.46 \mathrm{gk}$ & $17.17 \mathrm{k}$ & $19.56 \mathrm{fk}$ & $18.92 \mathrm{ik}$ \\
\hline & HL & $30.31 \mathrm{ab}$ & $25.34 \mathrm{~cd}$ & $25.23 \mathrm{~cd}$ & $19.84 \mathrm{fk}$ & $19.07 \mathrm{ik}$ & $20.32 \mathrm{ek}$ \\
\hline & HL+Ethyl. & $25.43 \mathrm{~cd}$ & $27.42 b c$ & $22.33 \mathrm{dj}$ & $19.91 \mathrm{ek}$ & $18.65 \mathrm{jk}$ & $17.69 \mathrm{k}$ \\
\hline & CL & $33.23 \mathrm{a}$ & $23.82 \mathrm{cf}$ & $23.12 \mathrm{di}$ & $19.90 \mathrm{ek}$ & $19.71 \mathrm{fk}$ & $16.18 \mathrm{k}$ \\
\hline & CL+Ethyl. & $30.20 \mathrm{ab}$ & $23.53 \mathrm{ch}$ & $24.10 \mathrm{ce}$ & $19.35 \mathrm{gk}$ & $19.23 \mathrm{hk}$ & $16.07 \mathrm{k}$ \\
\hline
\end{tabular}

*: Means with different letters are statistically significant at $\mathrm{P} \leq 0.05$ according to Duncan's multiple range test; ${ }^{\dagger} \mathrm{BS}$ : Beefsteak; BS+Ethyl.: Beefsteak+Ethylene, HL: Heirloom; HL+Ethyl.: Heirloom+Ethylene; CL: Cluster; CL+Ethyl.: Cluster+Ethylene; LSD: Least significant difference; St. Dur.: Storage duration, St. Dur. $\times$ Trt.: Storage duration $\times$ Treatments; Trt: Treatments 
Table 2- Effect of ethylene on the total chlorophyll, lycopene, total phenolic contents, antioxidant activity, carotenoid, flavonoid, and ascorbic acid contents of different types of tomatoes under shelf life conditions at $20^{\circ} \mathrm{C}$

\begin{tabular}{|c|c|c|c|c|c|c|c|}
\hline \multirow[b]{2}{*}{ Parameters } & \multirow[b]{2}{*}{ Treatments } & \multicolumn{6}{|c|}{ Storage duration (Days) } \\
\hline & & 0 & $7+3$ & $14+3$ & $21+3$ & $28+3$ & $35+3$ \\
\hline \multirow{7}{*}{$\begin{array}{l}\text { Total chlorophyll content } \\
\left(\mathrm{g} \mathrm{kg}^{-1} \text { fw) }\right.\end{array}$} & $\mathbf{B S}^{\dagger}$ & $0.0050 \mathrm{ad}$ & $0.0041 \mathrm{af}$ & $0.0036 \mathrm{ag}$ & $0.0034 \mathrm{ci}$ & $0.0018 \mathrm{ej}$ & $0.0016 \mathrm{fj}$ \\
\hline & BS+Ethyl. & $0.0038 \mathrm{ag}$ & $0.0036 \mathrm{ag}$ & $0.0025 \mathrm{dj}$ & $0.0020 \mathrm{ej}$ & $0.0020 \mathrm{ej}$ & $0.0015 \mathrm{fj}$ \\
\hline & HL & $0.0044 \mathrm{ae}$ & $0.0044 \mathrm{ae}$ & $0.0044 \mathrm{ae}$ & $0.0016 \mathrm{fj}$ & $0.0007 \mathrm{j}$ & $0.0005 \mathrm{j}$ \\
\hline & HL+Ethyl. & 0.0040af & $0.0043 \mathrm{ae}$ & 0.0036ag & $0.0024 \mathrm{ej}$ & $0.0006 \mathrm{j}$ & $0.0006 \mathrm{j}$ \\
\hline & CL & $0.0061 \mathrm{ab}$ & $0.0061 \mathrm{a}$ & $0.0035 \mathrm{bh}$ & $0.0013 \mathrm{il}$ & $0.0008 \mathrm{ij}$ & $0.0006 \mathrm{j}$ \\
\hline & CL+Ethyl. & $0.0053 \mathrm{ac}$ & $0.0015 f j$ & $0.0014 \mathrm{fj}$ & 0.0009hj & $0.0007 \mathrm{j}$ & $0.0004 \mathrm{j}$ \\
\hline & \multicolumn{7}{|c|}{ LSD5\%: St. Dur.: 0.0009 St. Dur. $\times$ Trt.: 0.0022 Trt.: 0.0009} \\
\hline \multirow{7}{*}{$\begin{array}{l}\text { Lycopene content } \\
\left(\mathrm{mg} \mathrm{kg}^{-1} \text { fw }\right)\end{array}$} & BS & $1.93 \mathrm{p}$ & $5.88 \mathrm{np}$ & $16.87 \mathrm{kn}$ & $19.81 \mathrm{~km}$ & $25.16 \mathrm{gl}$ & $37.18 \mathrm{dg}$ \\
\hline & BS+Ethyl. & $5.10 \mathrm{op}$ & $9.72 \mathrm{mp}$ & 21.99il & $25.33 \mathrm{gl}$ & $32.40 \mathrm{ej}$ & $37.31 \mathrm{dg}$ \\
\hline & HL & $2.98 \mathrm{p}$ & $23.93 \mathrm{hl}$ & $37.05 \mathrm{dg}$ & $37.72 \mathrm{df}$ & $38.85 \mathrm{df}$ & $54.94 \mathrm{ab}$ \\
\hline & HL+Ethyl. & $4.07 \mathrm{op}$ & $21.69 \mathrm{jl}$ & $28.37 \mathrm{fk}$ & $42.75 \mathrm{ce}$ & $42.83 \mathrm{ce}$ & $56.36 \mathrm{a}$ \\
\hline & CL & $2.31 \mathrm{p}$ & $21.75 \mathrm{jl}$ & $35.29 \mathrm{eh}$ & $39.51 \mathrm{df}$ & 44.11be & 48.71ad \\
\hline & CL+Ethyl. & $2.98 \mathrm{p}$ & 14.9810 & $33.83 \mathrm{ei}$ & $34.50 \mathrm{eh}$ & 39.90df & $53.85 \mathrm{ac}$ \\
\hline & \multicolumn{7}{|c|}{ LSD5\%: St. Dur.: 4.2219 St. Dur. $\times$ Trt.: 10.342 Trt.: 4.2219} \\
\hline \multirow{7}{*}{$\begin{array}{l}\text { Antioxidant Activity } \\
\left(\mathrm{g} \mathrm{kg}^{-1} \text { fw EC } \mathrm{EC}_{50}\right)\end{array}$} & BS & $1.53 \mathrm{a}$ & $0.96 \mathrm{bd}$ & $0.41 \mathrm{jn}$ & $0.87 \mathrm{be}$ & $0.72 \mathrm{dh}$ & $0.60 \mathrm{fl}$ \\
\hline & BS+Ethyl. & $1.39 \mathrm{a}$ & $0.98 \mathrm{bc}$ & $0.80 \mathrm{bf}$ & $0.89 \mathrm{be}$ & $0.67 \mathrm{ej}$ & $0.69 \mathrm{ei}$ \\
\hline & HL & 0.44in & $0.26 n$ & $0.34 \ln$ & $0.36 \ln$ & $0.38 \mathrm{kn}$ & $0.47 \mathrm{hn}$ \\
\hline & HL+Ethyl. & $0.30 \mathrm{mn}$ & $0.30 \mathrm{mn}$ & $0.47 \mathrm{hn}$ & $0.39 \mathrm{kn}$ & $0.51 \mathrm{gn}$ & $0.41 \mathrm{jn}$ \\
\hline & $\mathbf{C L}$ & $1.02 \mathrm{~b}$ & $0.69 \mathrm{ei}$ & $0.55 \mathrm{fm}$ & $0.66 \mathrm{ej}$ & $0.63 \mathrm{ek}$ & $0.81 \mathrm{bf}$ \\
\hline & CL+Ethyl. & $1.50 \mathrm{a}$ & $0.56 \mathrm{fm}$ & 0.46 in & $0.74 \mathrm{cg}$ & $0.81 \mathrm{bf}$ & $0.76 \mathrm{cg}$ \\
\hline & \multicolumn{7}{|c|}{ LSD5\%: St. Dur.: 0.0888 St. Dur. $\times$ Trt.: 0.2175 Trt.: 0.0888} \\
\hline \multirow{7}{*}{$\begin{array}{l}\text { Carotenoid content } \\
\left(\mathrm{g} \mathrm{kg}^{-1} \mathrm{fw}\right)\end{array}$} & BS & $0.0046 \mathrm{e}$ & $0.0072 \mathrm{de}$ & $0.0078 \mathrm{de}$ & $0.0118 \mathrm{de}$ & $0.0051 \mathrm{e}$ & $0.0279 \mathrm{be}$ \\
\hline & BS+Ethyl. & $0.0042 \mathrm{e}$ & $0.0048 \mathrm{e}$ & $0.0055 \mathrm{e}$ & $0.0149 \mathrm{ce}$ & $0.0384 \mathrm{ad}$ & $0.0449 \mathrm{ac}$ \\
\hline & HL & $0.0048 \mathrm{e}$ & $0.0061 \mathrm{e}$ & $0.0133 \mathrm{de}$ & $0.0192 \mathrm{be}$ & $0.0240 \mathrm{be}$ & $0.0290 \mathrm{be}$ \\
\hline & HL+Ethyl. & $0.0045 \mathrm{e}$ & $0.0094 \mathrm{de}$ & $0.0128 \mathrm{de}$ & $0.0122 \mathrm{de}$ & $0.0230 \mathrm{be}$ & $0.0660 \mathrm{a}$ \\
\hline & $\mathbf{C L}$ & $0.0020 \mathrm{e}$ & $0.0020 \mathrm{e}$ & $0.0041 \mathrm{e}$ & $0.0087 \mathrm{de}$ & $0.0098 \mathrm{de}$ & $0.0149 \mathrm{ce}$ \\
\hline & CL+Ethyl. & $0.0051 \mathrm{e}$ & $0.0048 \mathrm{e}$ & $0.0087 \mathrm{de}$ & $0.0099 \mathrm{de}$ & $0.0315 \mathrm{ad}$ & $0.0454 \mathrm{ab}$ \\
\hline & \multicolumn{7}{|c|}{ LSD5\%: St. Dur.: 0.0105 St. Dur. $\times$ Trt.: 0.0256 Trt.: 0.0105} \\
\hline \multirow{7}{*}{$\begin{array}{l}\text { Flavonoid content } \\
\left(\mathrm{mg} \mathrm{kg}^{-1} \mathrm{fw}\right)\end{array}$} & BS & $252.7 \mathrm{a}$ & $190.8 \mathrm{ac}$ & 112.7be & $87.3 \mathrm{ce}$ & $30.5 \mathrm{e}$ & $24.1 \mathrm{e}$ \\
\hline & BS+Ethyl. & $237.2 \mathrm{ab}$ & $55.7 \mathrm{ce}$ & $31.1 \mathrm{e}$ & $45.7 \mathrm{de}$ & 41.6de & $8.9 \mathrm{e}$ \\
\hline & HL & 113.5be & $96.9 \mathrm{ce}$ & $82.7 \mathrm{ce}$ & $43.7 \mathrm{de}$ & $26.1 \mathrm{e}$ & $19.3 \mathrm{e}$ \\
\hline & HL+Ethyl. & 106.0be & 51.6ce & 50.7ce & $49.9 \mathrm{ce}$ & $47.4 \mathrm{ce}$ & $44.3 \mathrm{de}$ \\
\hline & $\mathbf{C L}$ & $133.2 \mathrm{ae}$ & $128.5 \mathrm{ae}$ & 66.0ce & 41.8de & $21.4 \mathrm{e}$ & $11.4 \mathrm{e}$ \\
\hline & CL+Ethyl. & $183.5 \mathrm{ad}$ & $68.7 \mathrm{ce}$ & $43.2 \mathrm{de}$ & 42.0de & $22.4 \mathrm{e}$ & $12.3 \mathrm{e}$ \\
\hline & \multicolumn{7}{|c|}{ LSD5\%: St. Dur.: 48.2 St. Dur. $\times$ Trt.: 118.1 Trt.: 48.2} \\
\hline \multirow{7}{*}{$\begin{array}{l}\text { Total phenolic contents } \\
\left(\mathrm{mg} \mathrm{kg}^{-1} \text { GAE fw) }\right.\end{array}$} & BS & 26.0be & $23.4 \mathrm{cf}$ & 25.6be & $19.3 \mathrm{ej}$ & $24.8 \mathrm{ce}$ & $12.6 \mathrm{ij}$ \\
\hline & BS+Ethyl. & $24.2 \mathrm{cf}$ & 19.0ej & $23.2 \mathrm{cf}$ & $21.0 \mathrm{dh}$ & $22.5 \mathrm{cf}$ & $11.6 \mathrm{j}$ \\
\hline & HL & $33.4 \mathrm{ab}$ & $33.4 \mathrm{ab}$ & $28.2 \mathrm{bd}$ & 22.7bd & $20.1 \mathrm{di}$ & $12.5 \mathrm{ij}$ \\
\hline & HL+Ethyl. & $37.2 \mathrm{a}$ & $30.0 \mathrm{ac}$ & $37.2 \mathrm{a}$ & $22.3 \mathrm{cf}$ & $16.2 \mathrm{fj}$ & $12.3 \mathrm{ij}$ \\
\hline & $\mathbf{C L}$ & $21.0 \mathrm{dh}$ & $18.5 \mathrm{ej}$ & $18.5 \mathrm{ej}$ & $16.1 \mathrm{fj}$ & $13.5 \mathrm{gj}$ & $13.3 \mathrm{hj}$ \\
\hline & CL+Ethyl. & $23.1 \mathrm{cf}$ & $21.7 \mathrm{cg}$ & $17.5 \mathrm{ej}$ & $23.1 \mathrm{cf}$ & $13.7 \mathrm{gj}$ & $12.4 \mathrm{ij}$ \\
\hline & \multicolumn{7}{|c|}{ LSD5\%: St. Dur.: 2.8 St. Dur. × Trt.: 6.9 Trt.: 2.8} \\
\hline \multirow{7}{*}{ 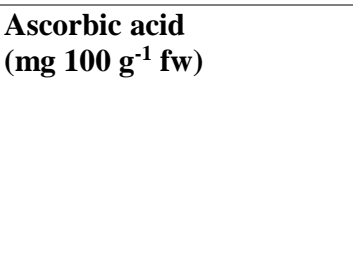 } & BS & $32.17 \mathrm{a}$ & $29.16 \mathrm{ac}$ & 25.31be & $19.57 \mathrm{fj}$ & $18.18 \mathrm{hk}$ & $13.96 \mathrm{kl}$ \\
\hline & BS+Ethyl. & $29.55 \mathrm{ab}$ & $25.48 \mathrm{bd}$ & $23.43 \mathrm{ch}$ & $19.56 \mathrm{fj}$ & 17.22il & 16.19il \\
\hline & HL & $30.31 \mathrm{ab}$ & $29.71 \mathrm{ab}$ & $23.83 \mathrm{cg}$ & 19.84ej & $21.25 \mathrm{di}$ & 17.05il \\
\hline & HL+Ethyl. & $25.43 \mathrm{bd}$ & $23.96 \mathrm{cf}$ & $21.17 \mathrm{di}$ & $20.37 \mathrm{di}$ & 19.91ej & 17.25il \\
\hline & $\mathbf{C L}$ & $33.23 \mathrm{a}$ & $25.56 \mathrm{bd}$ & $18.76 \mathrm{fk}$ & $19.86 \mathrm{ej}$ & $14.67 \mathrm{jl}$ & $17.35 \mathrm{il}$ \\
\hline & CL+Ethyl. & $30.20 \mathrm{ab}$ & $23.51 \mathrm{ch}$ & $20.97 \mathrm{di}$ & $18.49 \mathrm{gk}$ & $19.35 \mathrm{fj}$ & 12.541 \\
\hline & \multicolumn{7}{|c|}{ LSD5\%: St. Dur.: 1.82 St. Dur. $\times$ Trt.: 4.45 Trt.: 1.82} \\
\hline
\end{tabular}

*: Means with different letters are statistically significant at $\mathrm{P} \leq 0.05$ according to Duncan's multiple range test; 'BS; Beefsteak, BS+Ethyl.; Beefsteak+Ethylene; HL: Heirloom; HL+Ethyl.: Heirloom+Ethylene; CL: Cluster; CL+Ethyl.: Cluster+Ethylene; LSD: Least significant difference, St. Dur.: Storage duration; St. Dur. $\times$ Trt.: Storage duration $\times$ Treatments; Trt: Treatments.

During this study, the decline in chlorophyll content may be because of progress in ripening that caused color change in fruit from green to red maturity stage with the transformation of chloroplast into chromoplasts, breakdown of chlorophyll and synthesis of carotenoids occurred as reported by Alexander \& Grierson (2002). Watada et al. (1986) expressed that ethylene treatment enhances the degradation of chlorophyll in citrus fruit; turns it to yellow and then to orange color from green that agrees with the finding of less chlorophyll content obtained in this study in ethylene treated tomatoes. 


\subsection{Lycopene content}

Extending storage time had considerably increased the lycopene content in tomatoes. At the end of cold storage, the highest lycopene content $\left(47.37 \mathrm{mg} \mathrm{kg}^{-1}\right)$ was recorded in ethylene treated heirloom type while lowest lycopene content $\left(25.84 \mathrm{mg} \mathrm{kg}{ }^{-1}\right)$ was observed in control beefsteak type of tomatoes (Table 1). There were no significant differences between ethylene treated and control heirloom type of tomatoes at the end of cold storage. At the end of shelf life, the maximum lycopene content (56.36 $\left.\mathrm{mg} \mathrm{kg}^{-1}\right)$ was also recorded in ethylene treated heirloom type whereas the minimum lycopene content $\left(37.18 \mathrm{mg} \mathrm{kg}^{-1}\right)$ was noted in control beefsteak type of tomatoes (Table 2). However, there were no significant differences between ethylene treated and control heirloom type of tomatoes at the end of shelf life period.

Increase in lycopene concentration of tomato with extension in storage of tomato was reported by Khairi et al. (2015) which agreed with the findings in this study. Tadesse et al. (2016) reported as the maturity of tomato fruit at green mature stage enhances it converts chloroplast into chromoplast where the lycopene is present in membrane bound crystals. Dhall \& Singh (2013) reported higher lycopene content in ethylene treated tomatoes as obtained in this study. The lycopene content determined in the shelf life period were higher than cold storage in our experiment which was in confirmation with Tadesse et al. (2015) who expressed higher lycopene content in tomato kept at 20 and $30{ }^{\circ} \mathrm{C}$ than at $4{ }^{\circ} \mathrm{C}$.

\subsection{Antioxidant activity}

Antioxidant activity showed an increase in beefsteak and cluster types of tomatoes whereas in heirloom type of tomatoes it had decreased. There was no significant difference between control and ethylene treated cluster type of tomatoes on $35^{\text {th }}$ day of cold storage. The minimum $\mathrm{EC}_{50}$ value indicate the maximum antioxidant activity. After cold storage, the highest antioxidant activity $\left(0.33 \mathrm{~g} \mathrm{~kg}^{-1} \mathrm{EC}_{50}\right)$ was recorded in ethylene treated heirloom type while the lowest antioxidant activity $\left(0.89 \mathrm{~g} \mathrm{~kg}^{-1} \mathrm{EC}_{50}\right) \mathrm{was}$ noticed in ethylene treated beefsteak type of tomatoes (Table 1$)$. After shelf life period, the highest antioxidant activity $(0.41 \mathrm{~g}$ $\left.\mathrm{kg}^{-1} \mathrm{EC}_{50}\right)$ was recorded in ethylene treated heirloom type while the lowest antioxidant activity $\left(0.81 \mathrm{~g} \mathrm{~kg}^{-1} \mathrm{EC}_{50}\right)$ was observed in control cluster type of tomatoes (Table 2).

Tilahun et al. (2017) reported that antioxidant activity in tomatoes was higher at red maturity stage than those of harvested at green maturity stage which can be because of increase in lycopene concentration. This result confirmed our outcomes of ethylene treatment increased lycopene concentration and antioxidant activity.

\subsection{Carotenoid content}

Extending storage time had increased the carotenoid contents. Ethylene treated tomatoes had more carotenoid content than untreated ones. After cold storage, ethylene treated heirloom type tomatoes had maximum carotenoid content $\left(0.1182 \mathrm{~g} \mathrm{~kg}^{-1}\right)$ while minimum carotenoid content $\left(0.0172 \mathrm{~g} \mathrm{~kg}^{-1}\right)$ was noted in control heirloom type of tomatoes (Table 1$)$. At the end of shelf life period, maximum carotenoid content $\left(0.0660 \mathrm{~g} \mathrm{~kg}^{-1}\right)$ were also noted in ethylene treated heirloom type whereas minimum total carotenoid content $\left(0.0149 \mathrm{~g} \mathrm{~kg}^{-1}\right)$ was observed in control cluster type of tomatoes (Table 2).

In this study, ethylene treated tomatoes had higher carotenoid content at the end of storage which was in confirmation with the outcomes of Cruz et al. (2018). These researches expressed that ethylene regulates the carotenoid synthesis during ripening of tomatoes. Increase in carotenoid content in this study can be because of advancement in maturity that change color of tomato from green to red with conversion of chloroplast to chromoplast, resulting degradation of chlorophyll and accumulation of carotenoid as explained by Alexander \& Greirson (2002).

\subsection{Flavonoid content}

Flavonoid content decreased during storage. There was a significant interaction $(\mathrm{P} \leq 0.05)$ between storage duration and treatments. After cold storage, maximum flavonoid content $\left(42.2 \mathrm{mg} \mathrm{kg}^{-1}\right)$ occurred in ethylene treated heirloom fruit with minimum $\left(9.8 \mathrm{mg} \mathrm{kg}^{-1}\right)$ flavonoid content was in control beefsteak tomatoes (Table 1). During shelf life period, the highest flavonoid content $\left(44.3 \mathrm{mg} \mathrm{kg}^{-1}\right)$ was recorded in ethylene treated heirloom type with the lowest flavonoid content $\left(8.9 \mathrm{mg} \mathrm{kg}{ }^{-1}\right)$ was in ethylene treated beefsteak tomatoes (Table 2).

Riadh et al. (2016) stated that different cultivars of tomatoes significantly affected flavonoid content which agreed with significant effect obtained between different types of tomatoes treated with ethylene in our study. Flavonoid content showed decrease with increase in storage duration during our experiment which was supported by outcomes of Howard et al. (2000) who described decrease of flavonoid content during maturation of peppers. The losses in flavonoid content may be because of metabolic transformation to secondary phenolic compounds as reported by Barz \& Hoesel (1979). 


\subsection{Total phenolic contents}

Prolonging storage duration decreased the total phenolic contents. At the end of cold storage, the maximum total phenolic content (17.1 $\left.\mathrm{mg} \mathrm{kg}^{-1} \mathrm{GAE}\right)$ was exhibited by ethylene treated heirloom type whereas the minimum total phenolic contents $\left(9.0 \mathrm{mg} \mathrm{kg}{ }^{-1}\right.$ GAE) was found in control cluster type of tomatoes (Table 1). There were no significant differences between ethylene treated and control heirloom type of tomatoes by the end of cold storage. At the end of shelf life period, highest total phenolic content (13.3 $\left.\mathrm{mg} \mathrm{kg}^{-1} \mathrm{GAE}\right)$ was reported in control cluster type while lowest total phenolic content $\left(11.6 \mathrm{mg} \mathrm{kg}^{-1} \mathrm{GAE}\right)$ was recorded in ethylene treated beefsteak type of tomatoes (Table 2).

Extension in storage showed decrease in total phenolic contents of different types of tomatoes in our experiment. According to Day (2001) the higher respiration rate can be the reason of degradation of phenolic compounds. Control beefsteak type of tomatoes during cold storage had resulted more total phenolic contents which agreed with Dominguez et al. (2016) who demonstrated reduction in ethylene had increased the total phenolic contents in 'Delizia' tomato cultivar. The higher amount of total phenolic contents in heirloom type of tomatoes in this study during cold storage may be attributed to higher content of lycopene as described by Riadh et al. (2016).

\subsection{Ascorbic acid content}

Ascorbic acid displayed declining trend with extension in storage duration. At the end of cold storage, highest ascorbic acid content $\left(20.32 \mathrm{mg} 100 \mathrm{~g}^{-1}\right)$ was found in control heirloom type whereas lowest ascorbic acid content $\left(16.07 \mathrm{mg} 100 \mathrm{~g}^{-1}\right) \mathrm{was}$ reported in ethylene treated cluster type of tomatoes (Table 1). There were no significant differences between control beefsteak, ethylene treated heirloom, control cluster and ethylene treated cluster type of tomatoes by the end of cold storage. At the end of shelf life period, untreated cluster type of tomatoes had the maximum ascorbic acid contents $\left(17.35 \mathrm{mg}_{\left.100 \mathrm{~g}^{-1}\right)} \mathrm{while} \mathrm{the}\right.$ minimum ascorbic acid content (12.54 mg $100 \mathrm{~g}^{-1}$ ) was displayed by ethylene treated cluster type of tomatoes (Table 2). There were no significant differences between both ethylene treated and untreated heirloom and cluster type of tomatoes.

Declining trend in ascorbic acid content were exhibited by different types of tomatoes with extension in storage duration during this study which agreed with the findings of Tudor-Rado et al. (2016) who stated that decrease in ascorbic acid content of various tomato cultivars and the reason for decline in ascorbic acid may be because of oxidation caused by oxidizing enzymes. Our findings were in contradiction with Dhall \& Singh (2013) who reported ethylene treated tomatoes had more ascorbic acid content when comparison was made with control.

\section{Conclusions}

In conclusion, ethylene treatment resulted in higher lycopene and carotenoid contents with lower total chlorophyll contents in all tested tomato types. After cold storage and shelf life period, the maximum antioxidant activity, carotenoid and flavonoid content were recorded in ethylene treated heirloom type tomatoes. Furthermore, heirloom type tomatoes retained better postharvest quality as compared to beefsteak and cluster type of tomatoes at the end of 35 days of cold storage.

\section{Acknowledgements}

The financial support for this study was provided by Scientific Research Projects Coordination Unit of Akdeniz University. The authors would like to thank Prof. Errol W. Hewett for critical review of the manuscript.

\section{References}

Alexander L \& Grierson D (2002). Ethylene biosynthesis and action in tomato: a model for climacteric fruit ripening. Journal of Experimental Botany 53(377): 2039-2055. https://doi.org/10.1093/jxb/erf072

Barz W \& Hoesel W (1979). Metabolism and degradation of phenolic compounds in plants. Biochemistry of Plant Phenolics In: Swain T., Harbone J.B., Van Sumere C.F. (Eds.) Biochemistry of Plant Phenolics. Recent Advances in Phytochemistry, Springer, Boston, pp. 339369

Benvenuti S, Pellati F, Melegari M \& Bertelli D (2004). Polyphenols, anthocyanins, ascorbic acid, and radical scavenging activity of Rubs, Ribes and Aronia. Journal of Food Science 69(3): 164-169. https://doi.org/10.1111/j.1365-2621.2004.tb13352.x

Bhowmik D, Sampath Kumar K P, Paswan S \& Srivastava S (2012). Tomato-A natural medicine and its health benefits. Journal of Pharmacognosy and Phytochemistry 1(1): 33-43

Bosch M, Berger S, Schaller A \& Stintzi A (2014). Jasmonate-dependent induction of polyphenol oxidase activity in tomato foliage is important for defense against Spodoptera exigua but not against Manduca sexta. BMC Plant Biology 14: 257. https://doi.org/10.1186/s12870-0140257-8

Cemeroglu B (2010). Food Analysis, $2^{\text {nd }}$ edn. Publications of the Food Technology Society, Ankara (In Turkish)

Cruz A B, Bianchetti R E, Alves F R R, Purgato E, Peres L E P, Rossi M \& Freschi L (2018). Light, ethylene and auxin signaling interaction regulates, carotenoid biosynthesis during tomato fruit ripening. Frontiers in Plant Science 9: 1370. https://doi.org/10.3389/fpls.2018.01370

Day B P F (2001). Modified atmosphere packaging of fresh fruits and vegetables - an overview. Acta Horticulturae 553: 585-590. https://doi.org/10.17660/ActaHortic.2001.553.138 
Dhall R K \& Singh P (2013). Effect of ethephon and ethylene gas on ripening and quality of tomato (Solanum Lycopersicum L.) during cold storage. Journal of Nutrition and Food Sciences 3(6): 1-7. https://doi.org/10.4172/2155-9600.1000244

Dominguez I, Lafuente M T, Hernandez-Munoz P \& Gavara R (2016). Influence of modified atmosphere and ethylene levels on quality attributes of fresh tomatoes (Lycopersicon esculentum Mill.). Food Chemistry 209: 211-219. https://doi.org/10.1016/j.foodchem.2016.04.049

Fish W W, Perkins-Veazie P \& Collins J K (2002). A quantitative assay for lycopene that utilizes reduced volumes of organic solvents. Journal of Food Composition and Analysis 15(3): 309-317. https://doi.org/10.1006/jfca.2002.1069

Frusciante L, Carli P, Ercolano M R, Pernice R, Di Matteo A, Fogliano V \& Pellegrini N (2007). Antioxidant nutritional quality of tomato. Molecular Nutrition Food Research 51(5): 609-617. https://doi.org/10.1002/mnfr.200600158

George B, Kaur C, Khurdiya DS \& Kapoor HC (2004). Antioxidants in tomato (Lycopersicon esculentum) as a function of genotype. Food Chemistry 84: 45-51. https://doi.org/10.1016/S0308-8146(03)00165-1

Howard L R, Talcott S T, Brenes C H \& Villalon B (2000). Changes in phytochemical and antioxidant activity of selected pepper cultivars (Capsicum Species) as influenced by maturity. Journal of Agriculture and Food Chemistry 48(5): 1713-1720. https://doi.org/10.1021/jf990916t

Jimenez A, Creissen G, Kular B, Firmin J, Robinson S, Verhoeyen M \& Mullineaux P (2002). Changes in oxidative processes and components of the antioxidant system during fruit ripening. Planta 214: 751-758. https://doi.org/10.1007/s004250100667

Karadeniz F, Burdurlu H S, Koca N \& Soyer Y (2005). Antioxidant activity of selected fruit and vegetables grown in Turkey. Turkish Journal of Agriculture and Forestry 29(4): 297-303

Ke S-W, Chen G-H, Chen C-T, Tzen J T C \& Yang C-Y (2018). Ethylene signaling modulates contents of catechin and ability of antioxidant in Camelia sinensis. Botanical Studies 59: 11. https://doi.org/10.1186/s40529-018-0226-X

Khairi A N, Falah M A F, Suyatohadi A, Takahashi N \& Nishina H (2015). Effect of storage temperatures on color of tomato fruit (Solanum lycopersicum Mill.) cultivated under moderate water stress treatment. Agriculture and Agrícultural Science Procedia 3: 178-183. https://doi.org/10.1016/j.aaspro.2015.01.035

Lewis D R, Ramirez M V, Miller N D, Vallabhaneni P, Ray W K, Helm R F, Winkel B S J \& Muday G K (2011). Auxin and ethylene induce flavonol accumulation through distinct transcriptional networks. Plant physiology 156(1): 144-164. https://doi.org/10.1104/pp.111.172502

Lichtenthaler H K \& Wellburn A R (1983). Determination of total carotenoids and chlorophylls a and b of leaf extracts in different solvents. Biochemical Society Transactions 11(5): 591-592. https://doi.org/10.1042/bst0110591

Mcglasson W B (1970). The ethylene factor. In: AC Hulme (Ed), The biochemistry of fruits and their products, Academic Press, London, pp. 475-519

Nguyen M L \& Schwartz S J (1999). Lycopene: Chemical and biological properties. Food Technology 53(2): 38-45

Prasanna V, Prabha T N \& Tharanathan R N (2007). Fruit ripening phenomena-An overview. Critical Reviews in Food Science and Nutrition 47(1): 1-19. https://doi.org/10.1080/10408390600976841

Radzevicius A, Sakaluskiene S, Dagys M, Simniskis R, Karkleliene R, Bobinas C \& Duchovskis P (2013). The effect of strong microwave electric field radiation on: (1) vegetable seed germination and seedling growth rate. Zemdirbyte-Agriculture 100(2): 179-184. https://doi.org/10.13080/z-a.2013.100.023

Rao A V, Waseem Z \& Agarwal S (1998). Lycopene content of tomatoes and tomato products and their contribution to dietary lycopene. Food Research International 31(10): 737-741. https://doi.org/10.1016/S0963-9969(99)00053-8

Riadh I, Wasim S M, Imen T, Gabriela P, Salvatore L M \& Hdider C (2016). Functional quality and colour attributes of two high-lycopene tomato breeding lines grown under greenhouse conditions. Turkish Journal of Agriculture- Food Science and Technology 4(5): 365-373. http://doi.org/10.24925/turjaf.v4i5.365-373.620

Spanos G A \& Wrolstad R E (1990). Influence of processing and storage on the phenolic composition of Thompson seedless grape juice. Journal of Agricultural and Food Chemistry 38(7): 1565-1571. https://doi.org/10.1021/jf00097a030

Tadesse T N, Ibrahim A M \& Abtew W G (2015). Degradation and formation of fruit color in tomato (Solanum lycopersicum L.) in response to storage temperature. American Journal of Food Technology 10(4): 147-157. https://doi.org/10.3923/ajft.2015.147.157

Tadesse T N \& Abtew WG (2016). Effect of hot water treatment on reduction of chilling injury and keeping quality in tomato (Solanum lycopersicum L.) fruits. Journal of Stored Products and Postharvest Research 7(7): 61-68. https://doi.org/10.5897/JSPPR2016.0221

Tilahun S, Park D S, Taye A M \& Jeong C S (2017). Effects of storage duration on physicochemical properties of tomato (Lycopersicon esculentum Mill.). Horticultural Science and Technology 35(1): 88-97. https://doi.org/10.12972/kjhst.20170010

Tonucci L H, Holden J M, Beecher G R, Khachik F, Davis C S \& Mulokozi G (1995). Carotenoid content of thermally processed tomato-based food products. Journal of Agricultural and Food Chemistry 43: 579-586. https://doi.org/10.1021/jf00051a005

Tudor-Radu M, Vîjan L E, Tudor-Radu C M, Tita I, Sima R \& Mitrea R (2016). Assessment of ascorbic acid, polyphenols, flavonoids, anthocyanins and carotenoids content in tomato fruit. Notulae Botanicae Horti Agrobotanici Cluj-Napoca 44(2): 477-483. https://doi.org/10.15835/nbha44210332

Tucker G A (1993). Introduction. In: G Seymour, J Taylor \& G Tucker (Eds), Biochemistry of fruit ripening, Chapman \& Hall, London, pp. $1-51$

Viskelis P, Jankauskiene J \& Bobinaite R (2007). Influence of ripeness on tomato fruit quality. Sodininkyste ir Darzininkyste 26(4): 278-288

Witham F H, Blaydes D F \& Devlin R M (1971). Experiments in plant physiology. Van Nostrand Reinhold, New York

Watada A E (1986). Effects of ethylene on the quality of fruit and vegetables. Food Technology 40(5): 82-85

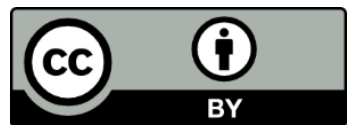

(C) 2022 by the author(s). Published by Ankara University, Faculty of Agriculture, Ankara, Turkey. This is an Open Access article distributed under the terms and conditions of the Creative Commons Attribution (CC BY) license (http://creativecommons.org/licenses/by/4.0/), which permits unrestricted use, distribution, and reproduction in any medium, provided the original work is properly cited. 